Copyright by the Acoustical Society of America. Song, O., Kwon, H. D., \& Librescu, L. (2001). Modeling, vibration, and stability of elastically tailored composite thin-walled beams carrying a spinning tip rotor. Journal of the Acoustical Society of America, 110(2), 877-886. doi: 10.1121/1.1377292

\title{
Modeling, vibration, and stability of elastically tailored composite thin-walled beams carrying a spinning tip rotor
}

\author{
Ohseop Song \\ Mechanical Engineering Department, Chungnam National University, Taejon 305-764, South Korea \\ Hyuck-Dong Kwon \\ Mechanical Engineering Department, Yonsei University, Seoul 120-749, South Korea \\ Liviu Librescua) \\ Engineering Science and Mechanics Department, Virginia Polytechnic Institute and State University, \\ Blacksburg, Virginia 24061
}

(Received 5 September 2000; revised 6 April 2001; accepted 14 April 2001)

\begin{abstract}
The problems of the mathematical modeling, eigenvibration, and stability of cantilevered thin-walled beams carrying a spinning rotor at its tip are investigated. The structure modeled as a thin-walled beam encompasses nonclassical features such as anisotropy, transverse shear, and secondary warping, and in this context, a special ply-angle configuration inducing a structural coupling between flapping-lagging-transverse shear is implemented. The implications of combined gyroscopic effects and conservative force upon the free vibration and stability of this structural system are revealed and a number of pertinent conclusions are outlined. Among others, it is shown that the judicious implementation of the tailoring technique can yield dramatic enhancements of both the vibrational and stability behavior of the system. (c) 2001 Acoustical Society of America. [DOI: $10.1121 / 1.1377292]$

PACS numbers: 43.40.At [CBB]
\end{abstract}

\section{INTRODUCTION}

A great deal of interest for the study of gyroscopic systems has been manifested in the last decade. This interest was stimulated by the need of a better understanding of the behavior of a number of important technological devices such as the drive shafts of gas turbines, of rotor systems used in helicopter and tilt rotor aircraft, as well as of robotic manipulators and gyro-devices used in space applications. Some of these robots carry tools, such as drills or grinding wheels that rotate. The extensive lists of references supplied in Refs. 1 and 2 reflect in full the interest afforded to the study of this problem. However, in the last years (see Refs. 3 and 4), a more encompassing concept of gyroscropic systems, referred to as gyroelastic ones, was developed. According to this concept, a gyroelastic system is constituted of a body considered to be continuous in mass, stiffness, and in gyricity, as well. Based on this concept, an efficient possibility of modeling elastic structures that are equipped with a large number of small spinning rotors spread over the structure can be devised. Moreover, within this concept, also the case of the continuum equipped with discrete spinning rotors can be accommodated. In several recent papers this concept was exemplified for the case of a Bernoulli-Euler solid beam containing a 1-D distribution of gyricity along the beam neutral axis and subjected to a conservative load (see, e.g., Ref. 4), whereas in Ref. 5 this concept was applied to the case of a solid beam carrying a spinning rotor located at the beam tip. In the present article, an encompassing structural model

a)Electronic mail: librescu@vt.edu equipped with a gyrodevice is considered. In this sense, an anisotropic thin-walled beam equipped at its free end with a small spinning rotor is considered. The beam model as considered in this article was developed in Refs. 6-8. In this context, one of the goals of this article is to model and analyze the vibrational behavior of the gyroelastic system (see Fig. 1) as a function of the spin rate $\Omega_{z}$ of the rotor and on the conservative compressive force acting along the longitudinal $z$ axis. Another goal of this research is to put into evidence the conditions under which, for the present gyroscopic system, the instabilities by divergence (i.e., the static one) and by flutter (i.e., the dynamic one) occur. In this context, it will be shown that the directionality property featured by the anisotropic materials of the structure can constitute important tools towards postponing the occurrence of such instabilities. To this end, in order to induce specific structural couplings beneficial for the structure, a circumferentially uniform stiffness configuration is considered. Within this ply-angle configuration the entire system of governing equations splits exactly into two independent systems, one of them involving the flapping-lagging-transverse shear coupling, and the other one involving the twist-extension coupling. For the problem at hand, only the case of the system undergoing the flappinglagging-transverse shear coupled motion will be considered.

\section{COORDINATE SYSTEMS. BASIC ASSUMPTIONS}

The case of a straight flexible thin-walled beam of length $L$ that is clamped at $Z=0$ and free at $Z=L$ where it carries a spinning rigid rotor of mass $m$ is considered (see 


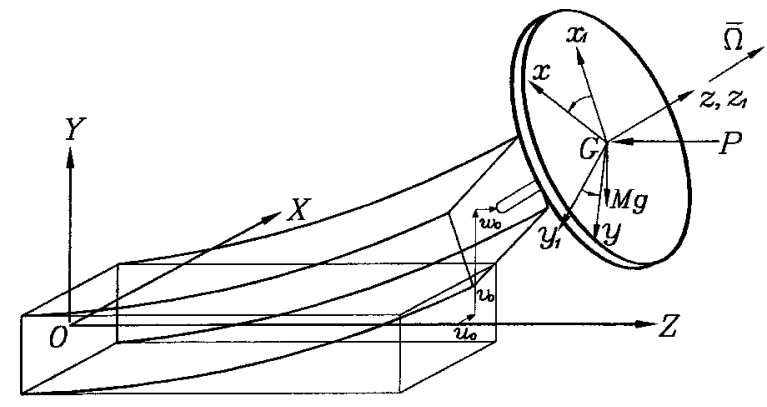

FIG. 1. Thin-walled box-beam with a spinning tip rotor.

Fig. 1). The offset between the beam tip and the centroid of the rotor is denoted as $r_{m}$. The rotor is assumed to have an axis of inertial symmetry which is coincident with the longitudinal axis of the beam.

Two sets of coordinates are considered, an inertial frame $O X Y Z$ and a body fixed frame Gxyz attached to the rotor, where $G$ is the rotor mass center. Associated to these two coordinate frames, the systems of the unit vectors are $\mathbf{I}, \mathbf{J}, \mathbf{K}$, and $\mathbf{i}, \mathbf{j}, \mathbf{k}$, respectively. The external force $P$ acting at the beam tip is assumed to remain parallel to the $Z$ axis of the inertial frame, whereas the spin rate vector of the rotor, $\boldsymbol{\Omega}_{z}$, is $\boldsymbol{\Omega}_{z}=\Omega_{z} \mathbf{k}$.

The adopted structural model is that of a thin-walled beam of arbitrary cross-sectional shape. Toward its modeling, the following assumptions are adopted: $(i)$ the original cross-section of the beam is preserved, (ii) the secondary warping effect is included, (iii) transverse shear effects are incorporated, and, finally, (iv) the constituent material of the structure features anisotropic properties, and, in this context, a special lay-up inducing flapping-lagging-transverse shear coupling is implemented. As a result, conditions preventing the occurrence of the twist motion should be implemented. Consequently, in this analysis the existence of the mass unbalance of the rotor is precluded.

\section{KINEMATICS}

In light of the previously mentioned assumptions and in order to reduce the 3-D elasticity problem to an equivalent $1-\mathrm{D}$ one, the components of the displacement vector are represented as in Refs. 1, 2 and 6-9 as

$$
\begin{aligned}
u(X, Y, Z ; t)= & u_{0}(Z ; t)-Y \phi(Z, t) \\
v(X, Y, Z ; t)= & v_{0}(Z ; t)+X \phi(Z, t) \\
w(X, Y, Z ; t)= & w_{0}(Z, t)+\theta_{X}(Z ; t)\left[Y(s)-n \frac{d X}{d s}\right] \\
& +\theta_{Y}(Z ; t)\left[X(s)+n \frac{d Y}{d s}\right] \\
& -\phi^{\prime}(Z ; t)\left[F_{\omega}(s)+n a(s)\right] .
\end{aligned}
$$

In these equations $u_{0}(Z ; t), v_{0}(Z ; t)$, and $w_{0}(Z ; t)$ denote the displacements along the $X, Y$ and $Z$ axes, respectively, while
$\phi(Z, t), \theta_{X}(Z ; t)$, and $\theta_{Y}(Z ; t)$ denote the twist about the $Z$ axis and rotations about the $X$ and $Y$ axes, respectively, $F_{\omega}(s)$ and $n a(s)$ that appear in the longitudinal displacement play the role of primary and secondary warping functions, respectively. For their definition see Refs. 6-8. In these equations, as well as in the forthcoming ones, the primes denote differentiation with respect to the longitudinal $Z$-coordinate, $s$ is the local circumferential coordinate measured along the mid-surface beam contour, while $n$ is the local normal coordinate measured from the mid-surface of the beam cross-section through the wall thickness. The rotational displacement of the beam tip can be expressed as $\Theta$ $=\left[\theta_{X},-\theta_{Y}, \phi\right]^{T}$, while the angular velocity $\Omega_{\mathbf{B}}$ of the beam cross-section where the rotor is attached is represented in the absolute system of coordinates through the symbolism of Hughes (see Ref. 10) as

$$
\begin{aligned}
\Omega_{B}=\Theta-\frac{1}{2} \Theta^{X} \dot{\Theta}= & {\left[\dot{\theta}_{X}+\frac{1}{2}\left(\theta_{Y} \dot{\phi}-\dot{\theta}_{Y} \phi\right) ;-\dot{\theta}_{Y}+\frac{1}{2}\left(\theta_{X} \dot{\phi}\right.\right.} \\
& \left.\left.-\dot{\theta}_{X} \phi\right) ; \dot{\phi}+\frac{1}{2}\left(\theta_{X} \dot{\theta}_{Y}-\dot{\theta}_{X} \theta_{Y}\right)\right]^{T} .
\end{aligned}
$$

In Eq. (2), $\Theta^{X}$ is the skew-symmetric matrix expressed in terms of the elements of the column matrix $\Theta$ as

$$
\Theta^{X}=\left|\begin{array}{ccc}
0 & -\phi & -\theta_{Y} \\
\phi & 0 & -\theta_{X} \\
\theta_{Y} & \theta_{X} & 0
\end{array}\right| .
$$

In addition, the superposed dots and superscript $T$ denote time derivatives and transpose of a matrix, respectively. Since $\Omega_{B}$ as expressed by Eq. (2) has to be evaluated at the beam tip, the terms associated with its effect appear in the boundary conditions at $Z=L$.

\section{GOVERNING EQUATIONS}

Toward the goal of deriving the equations of motion of beams equipped with a spinning rotor at its tip and the associated boundary conditions, Hamilton's variational principle is used. It may be stated as

$$
\delta J=\int_{t_{0}}^{t_{1}}\left(\delta U-\delta K_{B}-\delta K_{R}-\delta W_{R}-\delta W_{A}\right) d t=0,
$$

where $U$ denotes the strain energy of the elastic beam, $K_{B}$ and $K_{R}$ denote the kinetic energy of the beam, and of the spinning rotor, respectively, $W_{R}$ and $W_{A}$ denote the work done by spinning rotor and axial load, respectively, while $\delta$ denotes the variation operator.

In the forthcoming developments, in order to render explicitly the various energies intervening in Eq. (4), the relationship between the unit vectors $(\mathbf{I}, \mathbf{J}, \mathbf{K})$ and $(\mathbf{i}, \mathbf{j}, \mathbf{k})$ has to be established. To this end, after lengthy but straightforward manipulations one obtains the relationship

$$
\left(\begin{array}{c}
\mathbf{I} \\
\mathbf{J} \\
\mathbf{K}
\end{array}\right)=[H]\left(\begin{array}{c}
\mathbf{i} \\
\mathbf{j} \\
\mathbf{k}
\end{array}\right),
$$

where, by using the concept of small angles of rotation, we have 


$$
[H] \equiv h_{i j}=\left[\begin{array}{ccc}
\cos \Omega t-\phi \sin \Omega t & -(\sin \Omega t+\phi \cos \Omega t) & -\theta_{Y} \\
\sin \Omega t+\phi \cos \Omega t & \cos \Omega t-\phi \sin \Omega t & -\theta_{X} \\
\theta_{X} \sin \Omega t+\theta_{Y} \cos \Omega t & \theta_{X} \cos \Omega t-\theta_{Y} \sin \Omega t & 1
\end{array}\right] .
$$

By virtue of (2), the total angular velocity of the rotor can be expressed as

$$
\begin{aligned}
\boldsymbol{\Omega}_{R}= & \boldsymbol{\Omega}_{B}+\Omega_{z} \mathbf{k} \\
= & {\left[\dot{\theta}_{X}+\frac{1}{2}\left(\theta_{Y} \dot{\phi}-\dot{\theta}_{Y} \phi\right)\right] \mathbf{I}+\left[-\dot{\theta}_{Y}+\frac{1}{2}\left(\theta_{X} \dot{\phi}-\dot{\theta}_{X} \phi\right)\right] \mathbf{J} } \\
& +\left[\dot{\phi}+\frac{1}{2}\left(\theta_{X} \dot{\theta}_{Y}-\dot{\theta}_{X} \theta_{Y}\right)\right] \mathbf{K}+\Omega_{z} \mathbf{k} \\
= & \omega_{B X} \mathbf{I}+\omega_{B Y} \mathbf{J}+\omega_{B Z} \mathbf{K}+\Omega_{z} \mathbf{k} \\
= & \left(\omega_{B X} h_{11}+\omega_{B Y} h_{21}+\omega_{B Z} h_{31}\right) \mathbf{i}+\left(\omega_{B X} h_{12}+\omega_{B Y} h_{22}\right. \\
& \left.+\omega_{B Z} h_{32}\right) \mathbf{j}+\left(\omega_{B X} h_{13}+\omega_{B Y} h_{23}+\omega_{B Z} h_{33}+\Omega_{z}\right) \mathbf{k} \\
\equiv & \omega_{R x} \mathbf{i}+\omega_{R y} \mathbf{j}+\omega_{R z} \mathbf{k} .
\end{aligned}
$$

On the other hand, keeping in mind the expression of the position vector of the rotor

$\mathbf{R}_{R}(x, y, z ; t)=u_{0} \mathbf{I}+v_{0} \mathbf{J}+\left(L+w_{0}\right) \mathbf{K}+x \mathbf{i}+y \mathbf{j}+\left(r_{m}+z\right) \mathbf{k}$,

one obtains

$$
\dot{\mathbf{R}}_{R}=\dot{u}_{0} \mathbf{I}+\dot{v}_{0} \mathbf{J}+\dot{w}_{0} \mathbf{K}+x \dot{\mathbf{i}}+y \dot{\mathbf{i}}+z \dot{\mathbf{k}},
$$

wherefrom, by virtue of Eqs. (5) and (6), its expression becomes

$$
\begin{aligned}
\dot{\mathbf{R}}_{R}= & \dot{u}_{o} \mathbf{I}+\dot{v}_{o} \mathbf{J}+\dot{w}_{o} \mathbf{K}+\left(-y \omega_{R z}+z \omega_{R y}\right) \mathbf{i} \\
& +\left(x \omega_{R z}-z \omega_{R x}\right) \mathbf{j}+\left(-x \omega_{R y}+y \omega_{R x}\right) \mathbf{k} .
\end{aligned}
$$

As a result, it is possible to obtain the kinetic energy of the rotor as

$$
\begin{aligned}
K_{R}= & \frac{1}{2} \int_{\tau_{R}} \rho_{R}\left(\dot{\mathbf{R}}_{R} \cdot \dot{\mathbf{R}}_{R}\right) \delta_{\mathrm{D}}(Z-L) d \tau_{R} \\
= & \frac{1}{2}\left\{m_{R}\left(\dot{u}_{o}^{2}+\dot{v}_{o}^{2}+\dot{w}_{o}^{2}\right)+J_{x x y y}^{R}\left(\dot{\theta}_{X}^{2}+\dot{\theta}_{Y}^{2}\right)+J_{z z}^{R}\left[(\dot{\phi}+\Omega)^{2}\right.\right. \\
& \left.\left.+3 \Omega_{z}\left(\theta_{X} \dot{\theta}_{Y}-\dot{\theta}_{X} \theta_{Y}\right)\right]\right\}_{Z=L} .
\end{aligned}
$$

Herein, $\quad J_{x x}^{R}=J_{y y}^{R}=J_{x x y y}^{R}=m_{R}\left(r_{m}^{2}+\frac{1}{4} k_{R}^{2}\right) \quad$ are the rotor mass moments of inertia, $J_{z z}^{R}=\frac{1}{2} m_{R} k_{R}^{2}$ is the polar moment of inertia, $k_{R}$ is the radius of gyration of the rotor, and $m_{R}$ is the rotor mass, while $\delta_{\mathrm{D}}(\cdot)$ stands for the Dirac's delta function.

As a result, by using Hamilton's condition consisting of $\delta v_{i}=0$, at $t_{0}, t_{1}$, one obtains

$$
\begin{aligned}
\int_{t_{0}}^{t_{1}} \delta K_{R} d t \equiv & \left(\int_{t_{0}}^{t} d t \int_{\tau_{R}} \rho_{R} \ddot{\mathbf{R}}_{R} \cdot \delta \mathbf{R}_{R} d \tau_{R}\right) \\
= & {\left[-m_{R} \ddot{u}_{o} \delta u_{o}-m_{R} \ddot{v}_{o} \delta v_{o}-m_{R} \ddot{w}_{o} \delta w_{o}\right.} \\
& -\left(J_{x x y y}^{R} \ddot{\theta}_{X}-3 \Omega J_{z z}^{R} \dot{\theta}_{Y}\right) \delta \theta_{X}-\left(J_{x x y y}^{R} \ddot{\theta}_{Y}\right. \\
& \left.\left.+3 \Omega J_{z z}^{R} \dot{\theta}_{X}\right) \delta \theta_{Y}-J_{z z}^{R} \ddot{\phi} \delta \phi\right]_{Z=L} .
\end{aligned}
$$

As basic prequisites, we will list also the variation of the work done by the spinning tip rotor:

$$
\begin{aligned}
\delta W_{R}= & {\left[m_{R} r_{m} \ddot{\theta}_{Y} \delta u_{o}+m_{R}\left(r_{m} \ddot{\theta}_{X}-g\right) \delta v_{o}+m_{R} r_{m}\left(\ddot{v}_{o}+g\right)\right.} \\
& \left.\times \delta \theta_{X}+m_{R} r_{m} \ddot{u}_{o} \delta \theta_{Y}\right]_{Z=L}
\end{aligned}
$$

and also that done by the axial load

$$
\delta W_{A}=-P \int_{0}^{L}\left(u_{o}^{\prime \prime} \delta u_{o}+v_{o}^{\prime \prime} \delta v_{o}\right) d Z+P\left[u_{o}^{\prime} \delta u_{0}+v_{o}^{\prime} \delta v_{o}\right]_{0}^{L} .
$$

Finally, keeping in mind that the position vector and its time derivative of a deformed point of the beam are given, respectively, as

$$
\begin{aligned}
& \mathbf{R}_{b}(X, Y, Z ; t)=(X+u) \mathbf{I}+(Y+v) \mathbf{J}+(Z+w) \mathbf{K}, \\
& \dot{\mathbf{R}}_{b}(X, Y, Z ; t)=\dot{u} \mathbf{I}+\dot{v} \mathbf{J}+\dot{w} \mathbf{K},
\end{aligned}
$$

one can easily obtain the associated kinetic energy $K_{b}$ and also

$$
\begin{aligned}
\int_{t_{0}}^{t_{1}} \delta K_{b} d t \equiv & \left(-\int_{t_{0}}^{t_{1}} d t \int_{\tau} \ddot{\mathbf{R}}_{b} \cdot \delta \mathbf{R}_{b} d \tau\right) \\
= & -\int_{t_{0}}^{t_{1}} d t \int_{\tau}\left\{\left(\ddot{u}_{0}-Y \ddot{\phi}\right) \delta u+\left(\ddot{v}_{0}+X \ddot{\phi}\right) \delta v\right. \\
& +\left(\ddot{w}_{0}+\ddot{\theta}_{X}\left[Y-n \frac{d X}{d s}\right]+\ddot{\theta}_{Y}\left[X+\frac{d Y}{d s}\right]\right. \\
& \left.\left.-\ddot{\phi}^{\prime}\left[F_{w}+n a\right]\right) \delta w\right\} \rho d \tau \\
= & -\int_{t_{0}}^{t_{1}} d t\left[\int _ { 0 } ^ { L } \left\{K_{1} \delta u_{0}+K_{2} \delta v_{0}\right.\right. \\
& +K_{3} \delta w_{0} \delta \theta_{X}+K_{4} \theta_{X}+K_{5} \delta \theta_{Y} \\
& \left.\left.+\left(K_{6}-K_{7}\right) \delta \phi\right\} d Z-\left[K_{7} \delta \phi\right]_{0}^{L}\right] d t .
\end{aligned}
$$

In the above equation $K_{i}(i=\overline{1,7})$ denote inertia terms. Their expressions, restricted to circumferentially uniform 
stiffness configuration case that is considered in this article, are provided in Appendix A.

As concerns the variation of strain energy $U_{b}$ of the beam, its expression is

$$
\begin{aligned}
\delta U_{b}= & \frac{1}{2} \int_{\tau} \sigma_{i j} \delta \epsilon_{i j} d \tau \\
= & -\int_{0}^{L}\left\{Q_{X}^{\prime} \delta u_{0}+Q_{Y}^{\prime} \delta v_{0}+T_{Z} \delta w_{0}+\left(M_{X}^{\prime}-Q_{Y}\right) \delta \theta_{X}\right. \\
& \left.+\left(M_{Y}^{\prime}-Q_{X}\right) \delta \theta_{Y}+\left(B_{w}^{\prime \prime}+M_{Z}^{\prime}\right) \delta \phi\right\} d Z+\left[Q_{X} \delta u_{0}\right. \\
& +Q_{Y} \delta v_{0}+T_{A} \delta w_{0}+M_{x} \delta \theta_{X}+M_{Y} \delta \theta_{Y} \\
& \left.+\left(B_{w}^{\prime}+M_{Z}\right) \delta \phi-B_{w} \delta \phi^{\prime}\right]_{0}^{L}
\end{aligned}
$$

where $Q_{X}, Q_{Y}, T_{Z}, M_{X}, M_{Y}, M_{Z}$ and $B_{\omega}$ are 1-D stressresultants and stress-couples defined in Refs. 1, 6, and 7.

In addition, the variation of the work done by the gravitational force is

$$
\delta W_{b g}=-\int_{0}^{L} b_{1} g \delta v_{0} d Z,
$$

where $g$ is the gravitational acceleration while $b_{1}$ is a mass term whose expression is provided in Appendix A. In such a context, Hamilton's variational principle, Eq. (4), provides the equations of motion of the entire system and the associated boundary conditions. One of the main features of the obtained governing equation system is the full coupling between flapwise-bending, lateral (or chordwise) bending, extension, twist and transverse shear.

In order to induce the elastic coupling between flapwisebending, chordwise-bending and transverse shear, a special ply-angle distribution referred to as circumferentially uniform stiffness (CUS) configuration (see Refs. 6 and 7), generated by skewing angle plies with respect to the beam axis according to the law $\theta(Y)=\theta(-Y)$ and $\theta(X)=\theta(-X)$, is implemented.

The ply-angle configuration of this type is achievable via winding technology. Angle $\theta$ denotes the dominant ply orientation in the top and bottom, as well as in the lateral walls of the beam, measured from the positive $s$ axis toward the positive $Z$ axis.

In this case, from the variational equation (4) expressed in full, bearing in mind that $\delta u_{0}, \delta v_{0}, \delta \theta_{X}$ and $\delta \theta_{Y}$ are independent and arbitrary, and setting the coefficients of these variations to zero in the variational principle, the equations of motion and the boundary conditions featuring this type of coupling are obtained.

Employing constitutive equations and straindisplacement relationships in the equations of motion, the governing equations about a static equilibrium position featuring the flap-lag-transverse shear coupling expressed in terms of displacement quantities are obtained as

$$
\begin{aligned}
& \delta u_{o}: a_{43} \theta_{X}^{\prime \prime}+a_{44}\left(u_{o}^{\prime \prime}+\theta_{Y}^{\prime}\right)-b_{1} \ddot{u}_{o}-P u_{o}^{\prime \prime}=0, \\
& \delta v_{o}: a_{52} \theta_{Y}^{\prime \prime}+a_{55}\left(v_{o}^{\prime \prime}+\theta_{X}^{\prime}\right)-b_{1} \ddot{v}_{o}-P v_{o}^{\prime \prime}=0,
\end{aligned}
$$

$$
\begin{aligned}
& \delta \theta_{X}: a_{33} \theta_{X}^{\prime \prime}+a_{34}\left(u_{o}^{\prime \prime}+\theta_{Y}^{\prime}\right)-a_{55}\left(v_{o}^{\prime}+\theta_{X}\right)-a_{52} \theta_{Y}^{\prime} \\
& -\left(b_{4}+\delta_{n} b_{14}\right) \ddot{\theta}_{X}=0 \text {, } \\
& \delta \theta_{Y}: a_{22} \theta_{Y}^{\prime \prime}+a_{25}\left(v_{o}^{\prime \prime}+\theta_{X}^{\prime}\right)-a_{44}\left(u_{o}^{\prime}+\theta_{Y}\right)-a_{43} \theta_{X}^{\prime} \\
& -\left(b_{5}+\delta_{n} b_{15}\right) \ddot{\theta}_{Y}=0 \text {. }
\end{aligned}
$$

Associated to the governing system, the boundary conditions are at $Z=0$

$$
u_{o}=v_{o}=\theta_{X}=\theta_{Y}=0,
$$

and at $Z=L$,

$$
\begin{aligned}
& \delta u_{o}: a_{43} \theta_{X}^{\prime}+a_{44}\left(u_{o}^{\prime}+\theta_{Y}\right)-P u_{o}^{\prime}+m_{R}\left(\ddot{u}_{o}-r_{m} \ddot{\theta}_{Y}\right)=0 \text {, } \\
& \delta v_{o}: a_{52} \theta_{Y}^{\prime}+a_{55}\left(v_{o}^{\prime}+\theta_{X}\right)-P v_{o}^{\prime}+m_{R}\left(\ddot{v}_{o}-r_{\sim_{\sim}} \ddot{\theta}_{X}\right)=0 \text {, } \\
& \delta \theta_{X}: a_{33} \theta_{X}{ }^{\prime}+a_{34}\left(u_{o}^{\prime}+\theta_{Y}\right)+\underbrace{J_{\sim} y y}_{\sim \sim \sim} \ddot{\theta}_{\sim}-\underline{3 \Omega_{z} J_{z z}^{R} \dot{\theta}_{Y}} \\
& -m_{R} r_{m} \ddot{v}_{o}=0
\end{aligned}
$$

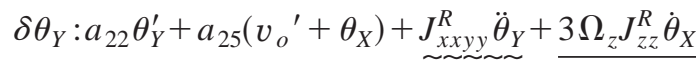

$$
\begin{aligned}
& -m_{R} r_{m} \ddot{u}_{o}=0 \text {. }
\end{aligned}
$$

The coefficients $a_{i j}=a_{j i}$ appearing in these equations as well as in the forthcoming ones denote stiffness quantities. Their expression can be found in Refs. 1 and 6-8. The terms in Eqs. (19) underscored by a solid line are associated with the gyroscopic effects, whereas those marked by an undulated line correspond to rotatory inertia terms. The tracer $\delta_{n}$ identifies the inertia effects associated to the points off the mid-surface of the beam. This tracer takes the value 1 or 0 depending on whether this effect is accounted for or discarded, respectively.

For locations of the spinning rotor different from that of the beam tip, the gryoscopic effects would have to appear in the governing equations and not in the boundary conditions at $Z=L$.

It should be remarked that for $\theta=0^{\circ}$ and $90^{\circ}$, the coupling stiffness quantities $a_{43}, a_{52}, a_{17}$ become immaterial, and when also $\Omega_{z}=0$, the governing system and boundary conditions split exactly into two independent groups associated with flapping and lagging motions. It should be mentioned that within the same ply-angle configuration an additional system of governing equations and boundary conditions involving the extension-twist coupling are obtained. Being decoupled from the system involving flapwisechordwise bending, these equations are not used in the article, and as such are not displayed here.

\section{THE CASE OF UNSHEARABLE BEAMS}

Equations (18)-(20), correspond to shearable beams. In order to determine their unshearable counterpart, elimination of $a_{44}\left(u_{0}^{\prime}+\theta_{Y}\right)$ and $a_{55}\left(v_{0}^{\prime}+\theta_{X}\right)$ in Eqs. (18a) and (18b) and in the boundary conditions (20a) and (20b) followed by consideration of $\theta_{X}=-v_{0}^{\prime}$ and $\theta_{Y}=-u_{0}^{\prime}$ results in the governing equations of the unshearable beams, 


$$
\begin{aligned}
& \left.\delta u_{o}: a_{22} u_{0}^{\prime \prime \prime}-\underset{\sim \sim}{\left(b_{5}+\delta_{n} b_{15}\right.}\right) \ddot{u}_{0}^{\prime \prime}+b_{1} \ddot{u}_{0}+P u_{0}^{\prime \prime}=0, \\
& \left.\delta v_{0}: a_{33} v_{0}^{\prime \prime \prime \prime}-\underset{\sim}{\left(b_{4}+\delta_{n} b_{14}\right.}\right) \ddot{v}_{0}^{\prime \prime}+b_{1} \ddot{v}_{0}+P v_{0}^{\prime \prime}=0,
\end{aligned}
$$

and the boundary conditions at $Z=0$,

$$
u_{o}=v_{0}=u_{0}^{\prime}=v_{0}^{\prime}=0 \text {, }
$$

and at $Z=L$,

$$
\begin{aligned}
& \delta u_{o}: a_{22} u_{0}^{\prime \prime \prime}-\left(b_{\sim} b_{\sim}+b_{15}\right) \ddot{u}_{0}^{\prime}+P u_{0}^{\prime}-m_{R}\left(\ddot{u}_{0}+r_{m \sim \sim} \ddot{v}_{0}^{\prime}\right)=0, \\
& \delta v_{0}: a_{33} v_{0}^{\prime \prime \prime}-\left(b_{\sim}^{b_{4}}+b_{14}\right) \ddot{v}_{0}^{\prime}+P v_{0}^{\prime}-m_{R}\left(\ddot{v}_{0}+r_{\sim_{\sim}} \ddot{v}_{0}^{\prime}\right)=0 \text {, } \\
& \delta u_{0}^{\prime}: a_{22} u_{0}^{\prime \prime}+J_{x x y y}^{R} \ddot{u}_{0}^{\prime}+3 \Omega_{z} J_{z z}^{R} \dot{v}_{0}^{\prime}+m_{R} r_{m} \ddot{u}_{0}=0 \text {, } \\
& \delta v_{0}^{\prime}: a_{33} v_{0}^{\prime \prime}+\underbrace{R}_{\text {xxyy }} \ddot{v}_{0}^{\prime}-3 \Omega_{z} J_{z z}^{R} \dot{u}_{0}^{\prime}+m_{R} r_{m} \ddot{v}_{0}=0 .
\end{aligned}
$$

In contrast to the case of shearable beams, it becomes evident that for the unshearable beam counterpart, the flapping-lagging coupling arises only in the boundary conditions at the beam tip, and when $r_{m}=0$, it is due entirely to the gyroscopic effects.

\section{SOLUTION METHODOLOGY}

Toward the goal of solving the eigenvalue problem of the gyroelastic system acted on by a compressive edge load applied at its tip, the following steps will be implemented. The first one consists of the representation of displacement quantities in the form

$$
\begin{aligned}
& u_{o}(Z ; t)=\mathbf{U}^{T}(Z) \mathbf{q}_{u}(t), \quad v_{o}(Z ; t)=\mathbf{V}^{T}(Z) \mathbf{q}_{v}(t), \\
& \theta_{X}(Z ; t)=\mathbf{X}^{T}(Z) \mathbf{q}_{x}(t), \quad \theta_{Y}(Z ; t)=\mathbf{Y}^{T}(Z) \mathbf{q}_{y}(t) .
\end{aligned}
$$

Herein $\mathbf{U}, \mathbf{V}, \mathbf{X}, \mathbf{Y}$ are the vectors of trial functions that are chosen as to satisfy at least the kinematic boundary conditions; $\mathbf{q}_{u}, \mathbf{q}_{v}, \mathbf{q}_{x}, \mathbf{q}_{y}$ are the vectors of generalized coordinates, while superscript $T$ denotes transpose operation. Replacing the energy quantities and representations (24) in the variational integral, Eq. (4), carrying out the indicated variations and the required integrations, leads to the equation

$$
\mathbf{M} \ddot{\mathbf{q}}(t)+\mathbf{G} \dot{\mathbf{q}}(t)+\mathbf{K q}(t)=\mathbf{0},
$$

where $\mathbf{M}$ is the real, symmetric, positive definite mass matrix, $\mathbf{K}$ is the symmetric stiffness matrix, including in its elements the external load $P, \mathbf{G}$ is the skew-symmetric gyroscopic matrix, while $\mathbf{q} \equiv\left[\mathbf{q}_{u}^{T}, \mathbf{q}_{v}^{T}, \mathbf{q}_{x}^{T}, \mathbf{q}_{y}^{T}\right]^{T}$ is the overall vector of the generalized coordinates. Notice that in the case of the nonspinning rotor, i.e., when $\Omega_{z}=0$, in the absence of structural damping the matrix $\mathbf{G}$ becomes immaterial. The expressions of the matrices $\mathbf{M}, \mathbf{K}$, and $\mathbf{G}$ are supplied in Appendix B.

For synchronous motion, upon expressing $\mathbf{q}(t)$ $=\mathbf{Z} \exp (i \omega t)$, where $\mathbf{Z}$ is a constant vector and $\omega$ is a constant valued quantity, both generally complex, and following the usual steps, one obtains the eigenvalue problem

$$
-\omega^{2} \mathbf{M Z}+i \omega \mathbf{G Z}+\mathbf{K Z}=\mathbf{0},
$$

where $\omega$ must satisfy the characteristic equation

$$
\Delta\left(\omega^{2}, P, \Omega_{z}\right)=\operatorname{det}\left|-\omega^{2} \mathbf{M}+i \omega \mathbf{G}+\mathbf{K}\right|=0 .
$$

In this connection we have to remark that the characteristic polynomial $\Delta$ contains only even powers of $\omega$ and, as such, the stability will be analyzed in terms of the nature of $\omega^{2}$. Since $\mathbf{K}$ includes the contribution of the external load which can be tensile or compressive, we can have $\mathbf{K}>0, \mathbf{K}=0$ or $\mathbf{K}<0$. As a result, one can distinguish three cases (see Ref. 11):

(1) Pure oscillatory motion, i.e., stable motion, occurring when $\mathbf{K}$ is positive definite, as long as $\omega_{k}^{2}$ are real and positive.

(2) Instability by divergence is found from the equation $\Delta\left(\omega^{2}, P\right)=0$ when $\omega^{2}=0$. As clearly emerges, the instability by divergence depends on the external load $P$ only and is the same for both the gyroscopic and nongyroscopic systems.

(3) $\mathbf{K}$ is negative definite. In this case it is still possible to have stable motion. In such a case the gyroscopic effects stabilize the unstable conservative system. However, also in the case, when $\mathbf{K}$ is negative definite, the eigenvalues can be complex conjugate with at least one of them having a negative imaginary part, which results in unstable motion of the flutter type. As the rotational speed of the rotor $\Omega_{z}$ and the compressive load $P$ increase, two consecutive eigenvalues $\omega_{p}^{2}$ and $\omega_{p}^{2}+1$ may coalesce, and beyond that point of coalescence, the eigenfrequencies become complex conjugate, and correspondingly, bending oscillations with exponentially increasing amplitudes will occur. The values of $\omega$ and $\Omega_{z}$ corresponding to the coalescence point are referred to as the flutter frequency $\omega_{f l}$, and the flutter rotational speed $\left(\Omega_{z}\right)_{f l}$, respectively. Certainly, at each magnitude of the external load $P, \omega_{f l}$ and $\left(\Omega_{z}\right)_{f l}$ can be determined. Although this type of instability is typical to nonconservative systems, this can occur in conservative gyroscopic systems as well.

\section{NUMERICAL SIMULATIONS}

The numerical illustrations are carried out for the case a composite box-beam. It features the following dimensions: $c=10$ in., $h=0.4$ in., $L=80$ in. It is also assumed that the material of the beam structure is of a Graphite/Epoxy composite whose on-axes elastic properties can be found in Refs. 1 and $6-8$.

The numerical simulations are displayed in terms of the following dimensionless parameters of the system:

$$
\begin{aligned}
& \overline{\omega_{i}}=\frac{\omega_{i}}{\omega_{0}}, \quad \bar{\Omega}_{z}=\frac{\Omega_{z}}{\omega_{o}}, \quad R=\frac{b}{c} ; \quad \bar{P}=\frac{\bar{P}}{\hat{P}}, \\
& \bar{m}_{R}=\frac{m_{R}}{b_{1} L}=0.5, \quad \bar{r}_{m}=\frac{r_{m}}{L}=0.2, \quad \bar{k}_{R}=\frac{k_{R}}{L}=0.2 .
\end{aligned}
$$

Herein $\omega_{0}=164.73 \mathrm{rad} / \mathrm{s}$ while $\hat{P}=81178.1 \mathrm{lb}$.

In Figs. 2(a)-4(a) there are depicted, in the absence of the compressive load $\bar{P}$, the dependence of the three normalized eigenfrequencies $\bar{\omega}_{i}(i=1,2,3)$ versus the normalized rotor spin speed $\bar{\Omega}_{z}$. The results were generated for a square cross-section $(R=1)$, unshearable beam, and for selected 

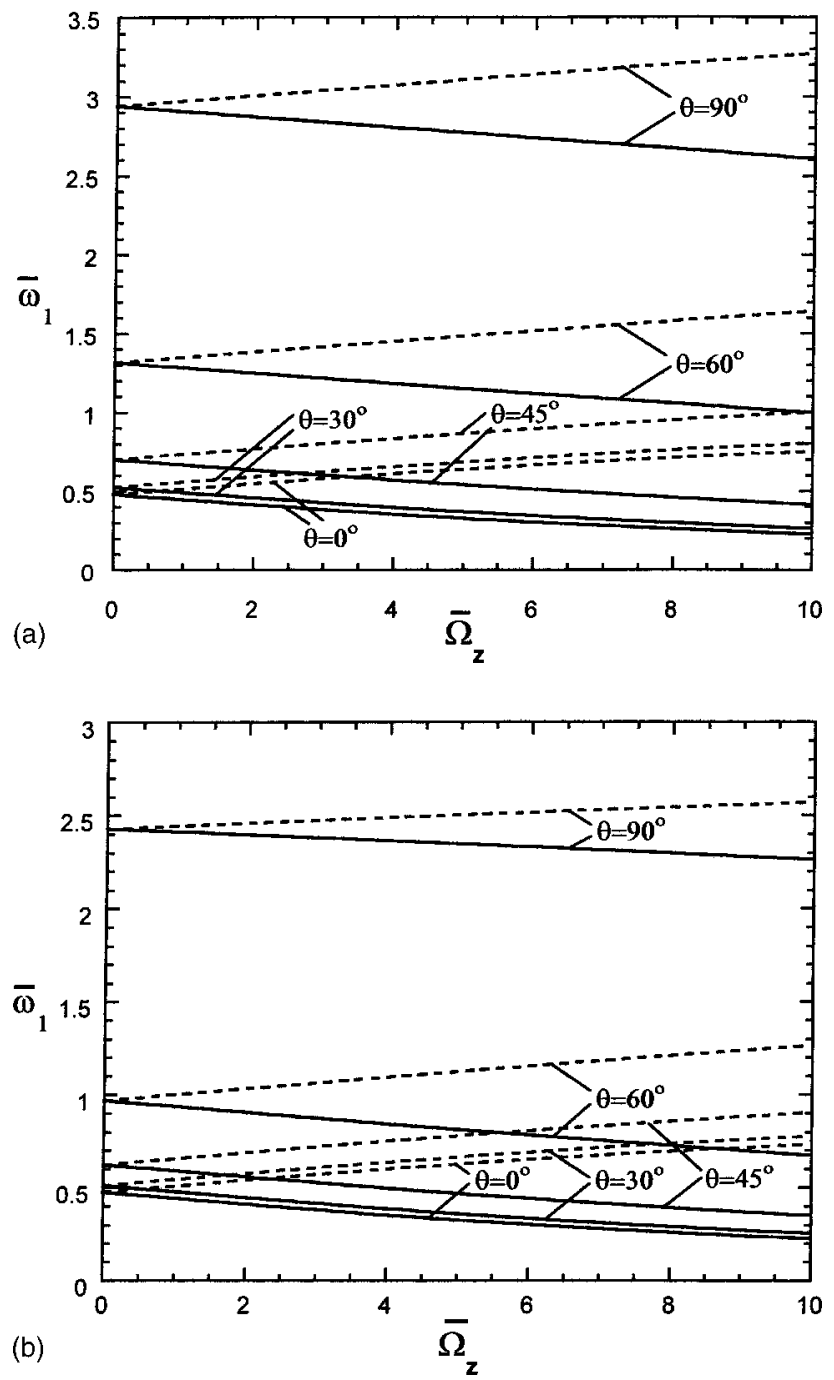

FIG. 2. (a) Influence of the ply-angle of the beam material on the upper and lower branches of the rotating dimensionless fundamental frequency. Unshearable beam model $R=1$. (b) The counterpart of (a) for the shearable beam model.

values of the ply-angle $\theta$. For $\bar{\Omega}_{z}=0$, i.e., for the nonspinning rotor, having in view that for this case the bending stiffnesses in $X$ and $Y$ directions are equal, the flapping and lagging eigenfrequencies in each mode coincide. As soon as the rotation starts, i.e., for $\bar{\Omega}_{z} \neq 0$, implying that gyroscopic forces are generated, a bifurcation of rotating eigenfrequencies is experienced, resulting in the upper and lower frequency branches. The rotor spin rate at which the lowest rotating eigenfrequency becomes zero-valued is referred to as the critical spinning speed, $\left(\bar{\Omega}_{z}\right)_{c r}$, and corresponds to the divergence instability. However, as it will appear later in Fig. 5, this critical spinning speed is extremely large. From these plots it can readily be seen that implementation of the tailoring technique can yield a dramatic enhancement of the vibrational behavior of the system. In this sense, the increase of the ply-angle $\theta$, which is accompanied by an increase of bending stiffnesses (see Ref. 7), results in an increase of both nonrotating and rotating eigenfrequencies.

From the same figure it clearly appears that, with the increase of the mode number, for fixed spin rates the dis-
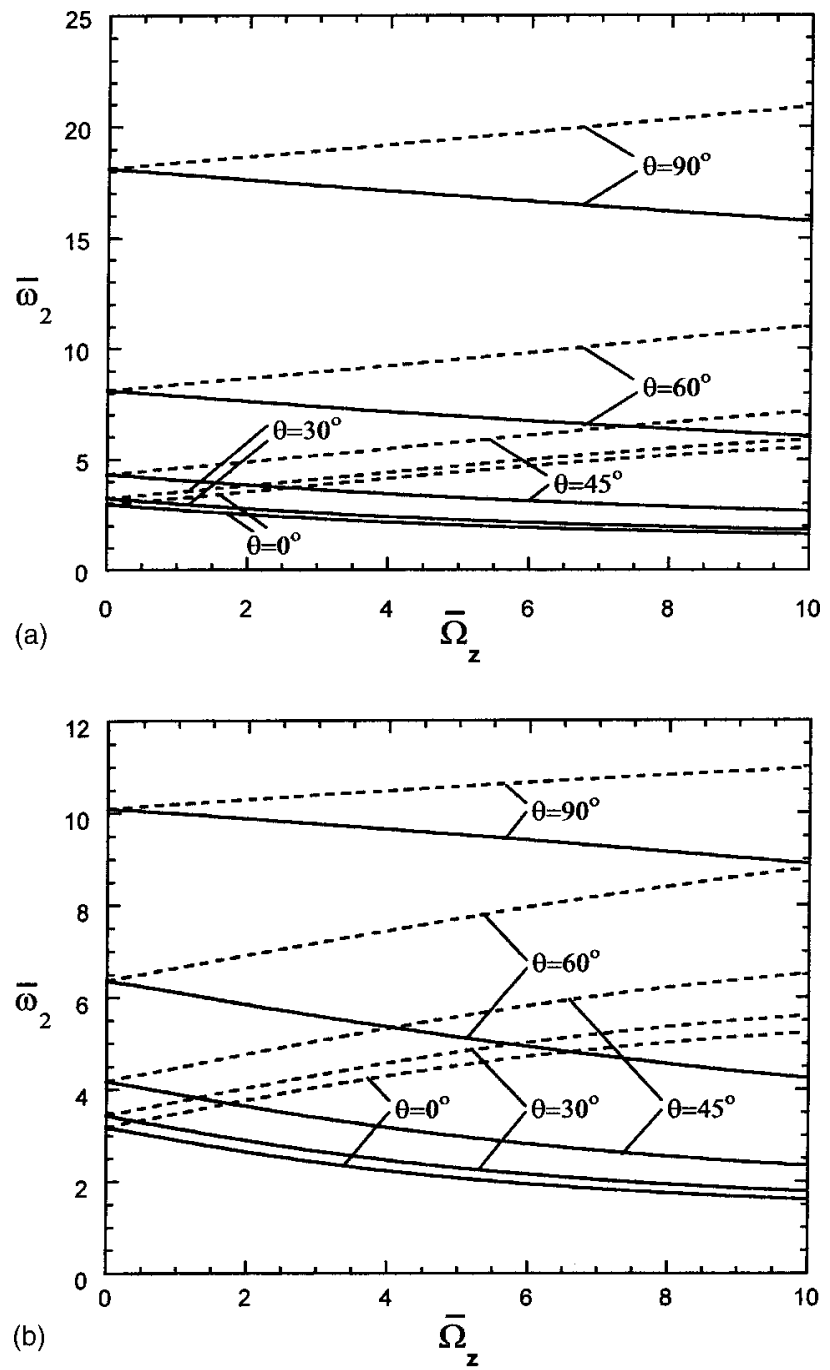

FIG. 3. (a) The counterpart of Fig. 2(a) for the second mode natural dimensionless frequency. (b) The counterpart of (a) for the shearable beam model.

tances between the upper and lower frequency branches as compared to those appearing in the lower modes tend to reduce in magnitude, a trend that is independent of the variation of the ply-angle. In Figs. 2(b)-4(b) the shearable counterparts of Figs. 2(a)-4(b) are depicted. Comparison of these figures reveals that the classical (unshearable) beam model overestimates both the nonrotating and the rotating frequencies.

In Fig. 5 the variation of the lower branch of the first eigenfrequency of the system versus the rotor spinning rate $\bar{\Omega}_{z}$ is depicted in logarithmic scale, for selected values of the ply-angle. The beneficial effects induced by the increase of the ply-angle on both the lower branch of rotating fundamental eigenfrequency and on the rotor spinning divergence speed clearly emerge from this plot. The same trend appears also in the case of the variation of the cross-section parameter $R$, in the sense that the increase of $R$ yields an increase of both the rotating/nonrotating lower branch of eigenfrequencies and of the divergence spinning speed of the rotor.

In Figs. 6-8 there are plots depicting the variation of the first three eigenfrequencies versus $\bar{\Omega}_{z}$ for selected values of the cross-sectional parameter $R$, and for a fixed value of the 

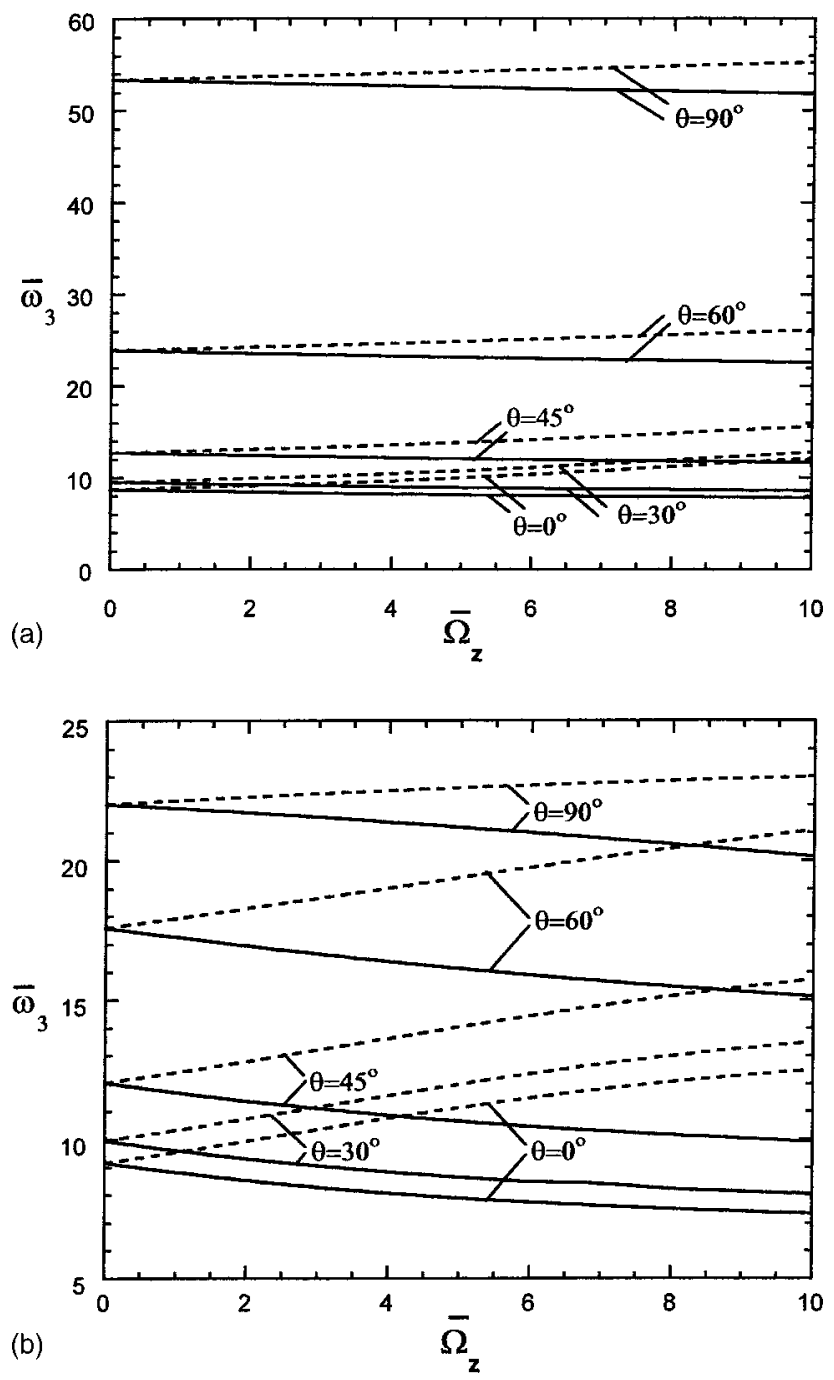

FIG. 4. (a) The counterpart of Fig. 2(a) for the third mode natural dimensionless frequency. (b) The counterpart of (a) for the shearable beam model.

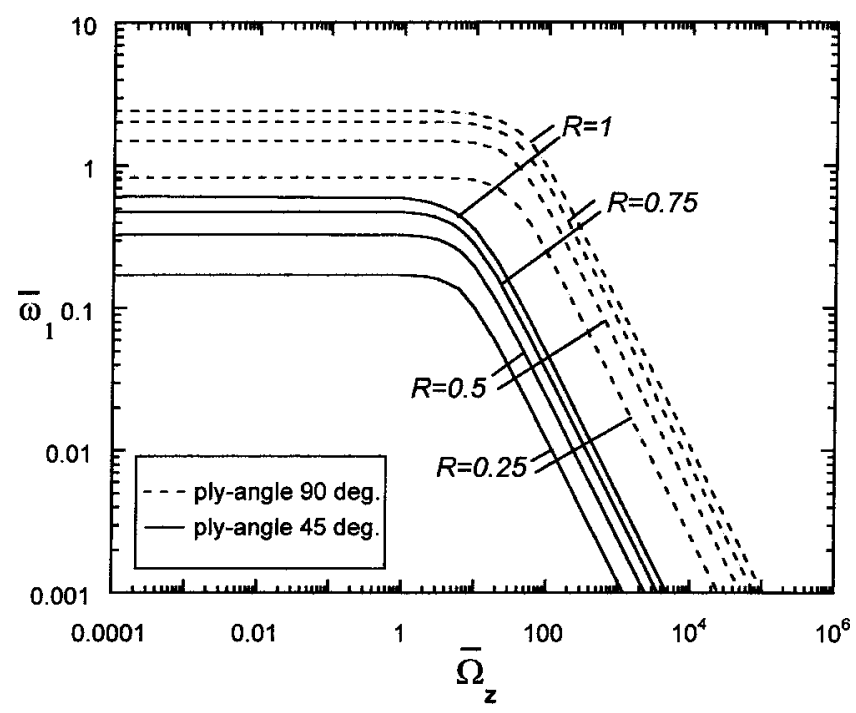

FIG. 5. Variation of the lower branch of the first natural rotating frequency for selected values of the cross-section parameter $R$, and two values of the ply-angle, $\theta=45$ and 90 degrees.

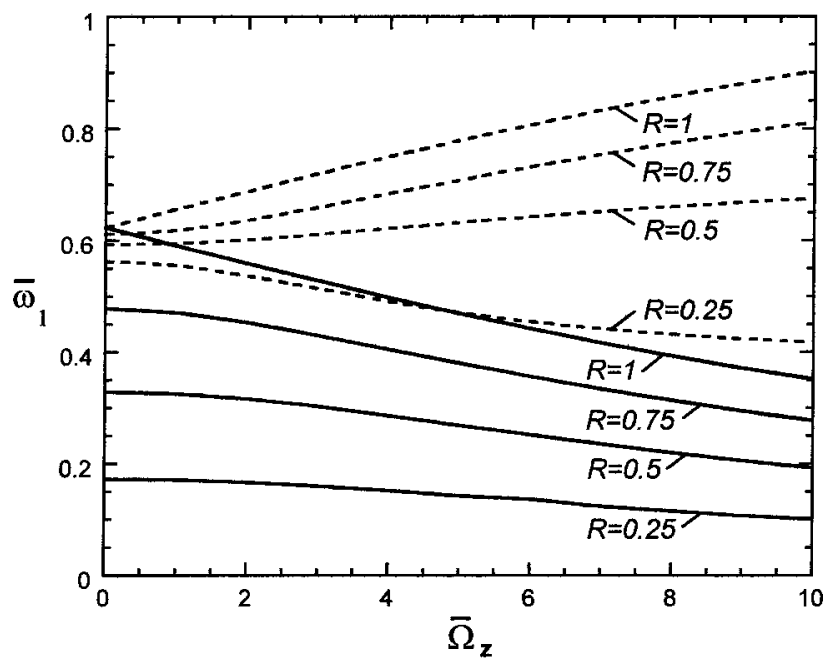

FIG. 6. Influence of the cross-section parameter $R$ on the upper and lower branches of the first natural dimensionless rotating frequency, $\theta=45$ degrees.

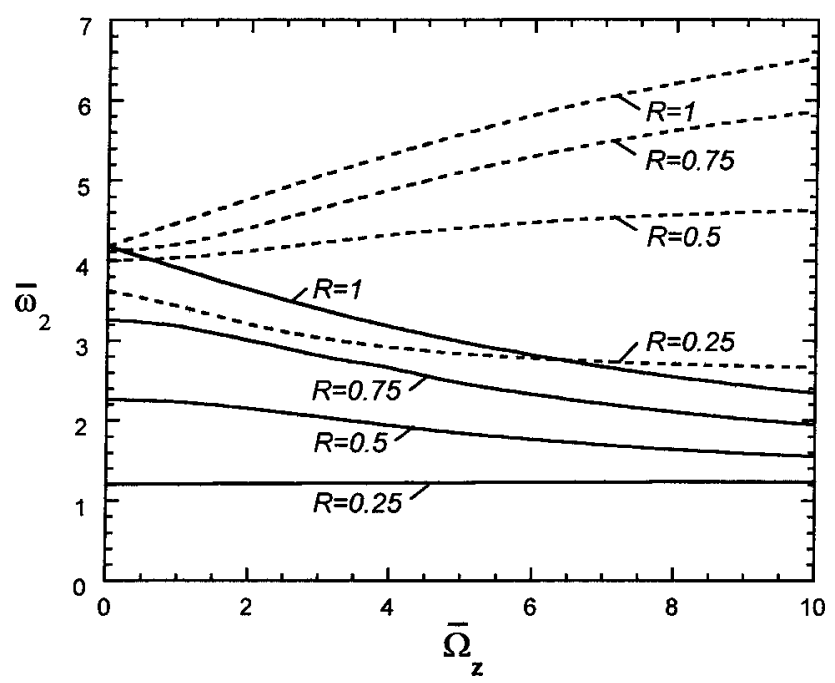

FIG. 7. Counterpart of Fig. 6 for the second rotating natural frequency.

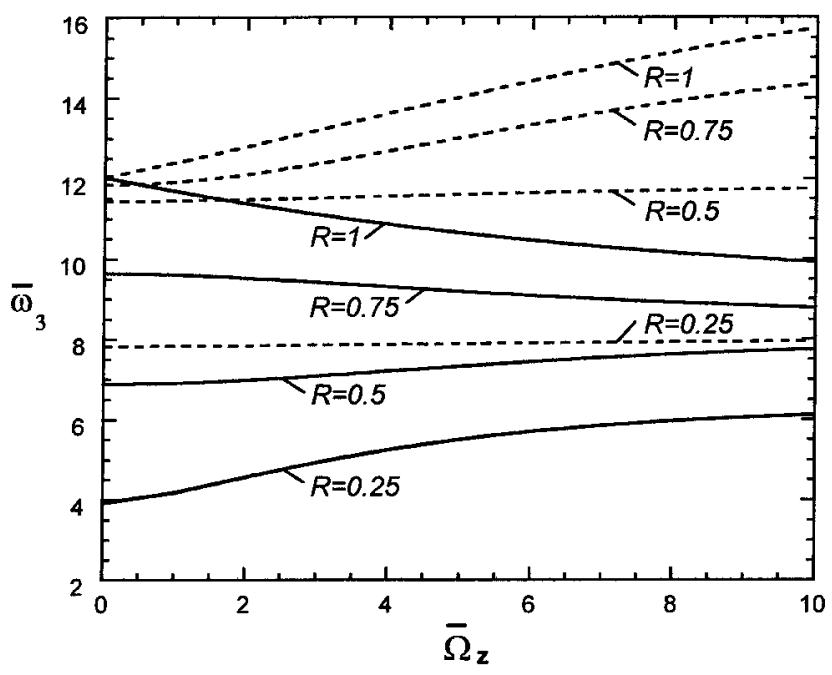

FIG. 8. Counterpart of Fig. 6 for the third rotating natural frequency. 


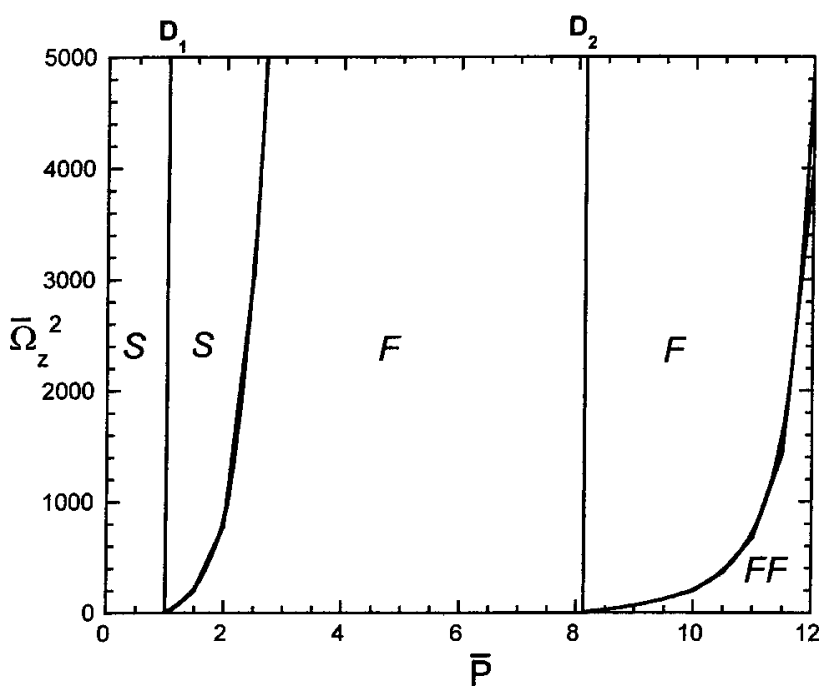

FIG. 9. Stability boundaries for the box-beam with a spinning rotor $(R$ $=1, \theta=0)$.

ply-angle $\theta=45$ degrees. The figures reveal that for beams characterized by $R \neq 1$, the nonrotating frequencies in flapping and lagging do not coincide.

It clearly appears that the natural frequency pairs associated to the case $R \neq 1$ are lower as compared to the ones associated to a square beam cross-section counterpart.

Moreover, with the increase of the mode number, the eigenfrequencies associated to the lower branch of each frequency pair, instead of decreasing, have the tendency of increasing with the increase of $\bar{\Omega}_{z}$, a trend that is more prominent for lower values of the parameter $R$.

In Figs. 9-11 stability plots for the case of a square $(R=1)$ cross-section beam are supplied. These were generated by representing the trial functions in Eqs. (24) by sixfunction approximation. In these figures the domains of stability are marked by $S$, and those by single and double flutter instability by $F$ and $F F$, respectively. For this case, since the beam features equal bending stiffnesses in both $X$ and $Y$ directions, the divergence instability under the compressive axial load occurs simultaneously in both $X$ and $Y$ directions.

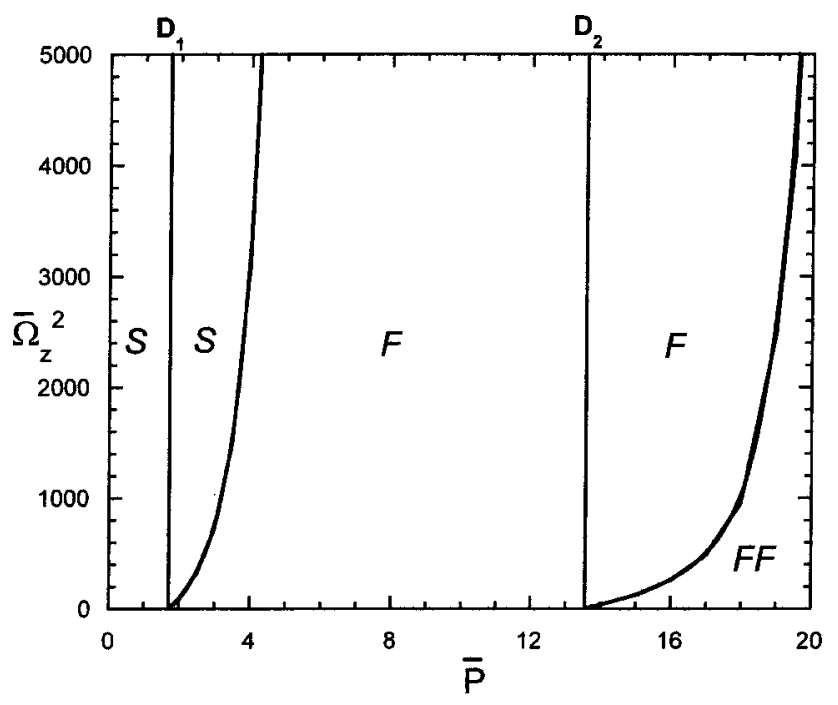

FIG. 10. Counterpart of Fig. 9 for $\theta=45$ degrees.

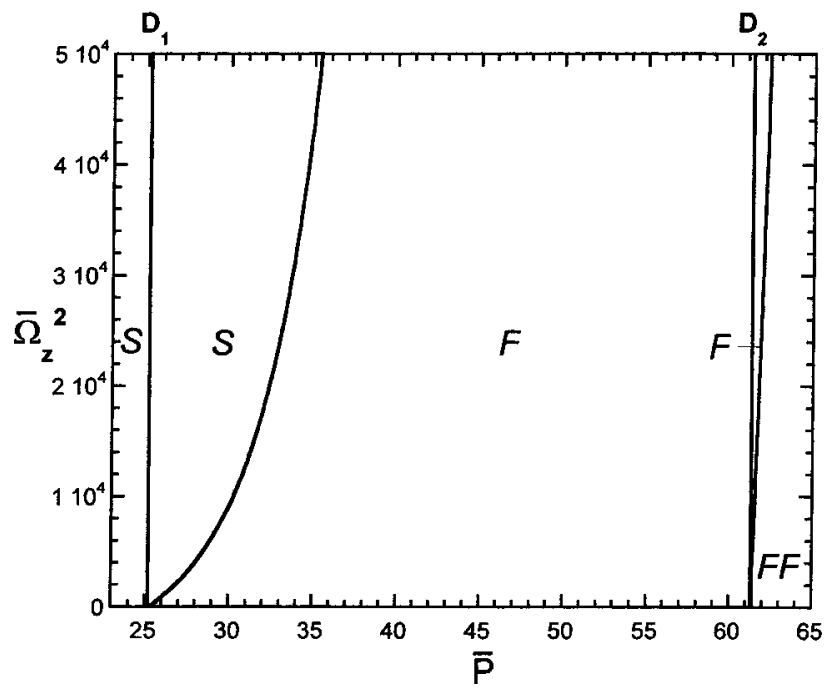

FIG. 11. Counterpart of Fig. 9 for $\theta=90$ degrees.

In this case the divergence boundaries marked as $D_{1}$ and $D_{2}$ are associated with double roots, and consequently these can be interpreted as divergence regions of zero thickness.

In the load interval $0 \leqslant \bar{P}<\bar{P}_{D_{1}}$, the frequencies $\omega_{k}^{2}$ remain positive for any value of $\bar{\Omega}_{z}^{2}$, indicating that in this load range the system is stable.

For a slight increase of $\bar{P}$ beyond $\bar{P}_{D_{1}}$ and for low values of $\bar{\Omega}_{z}^{2}$, a pair of complex conjugate eigenfrequencies indicating instability by single flutter is observed. However, as $\bar{\Omega}_{z}^{2}$ increases, due to the gyroscopic effects, the system featuring real positive roots $\omega_{k}^{2}$ enters into a stable region. One can interpret this behavior as the stabilization of a conservative system by gyroscopic forces.

However, at the right of the resulting stability boundary, until $P=\bar{P}_{D_{2}}$ and for arbitrary $\bar{\Omega}_{z}^{2}$, the frequencies consist of two positive values and a pair of complex conjugates indicating instability by single flutter. For $\bar{P}>\bar{P}_{D_{2}}$, two flutter regions are featured.

In one of these, the frequencies consist of two positive values and a pair of complex conjugates, indicating instability by single flutter (region $F$ ), while in another one, frequencies consist of two pairs of complex conjungates, indicating instability by double flutter (region $F F$ ).

From Figs. 9-11 one can infer that with the increase of the ply-angle the stability domains are greatly enlarged and, at the same time, the instability boundaries by divergence and flutter are shifted towards larger compressive loads $\bar{P}$.

It should be remarked that the stability behavior of the beam modeled within the thin-walled beam theory, as reported here, agrees qualitatively well with that obtained within the solid isotropic beam modeled within BernoulliEuler theory (see Ref. 5).

As concerns the stability of rectangular cross-section $(R \neq 1)$ thin-walled beams, their behavior is much more complex than that featured by their square cross-section beam counterparts, in the sense that in addition to regions corresponding to instability regions by single flutter $(F)$ and divergence $(D)$, regions by double $(D D)$, triple $(D D D)$, 


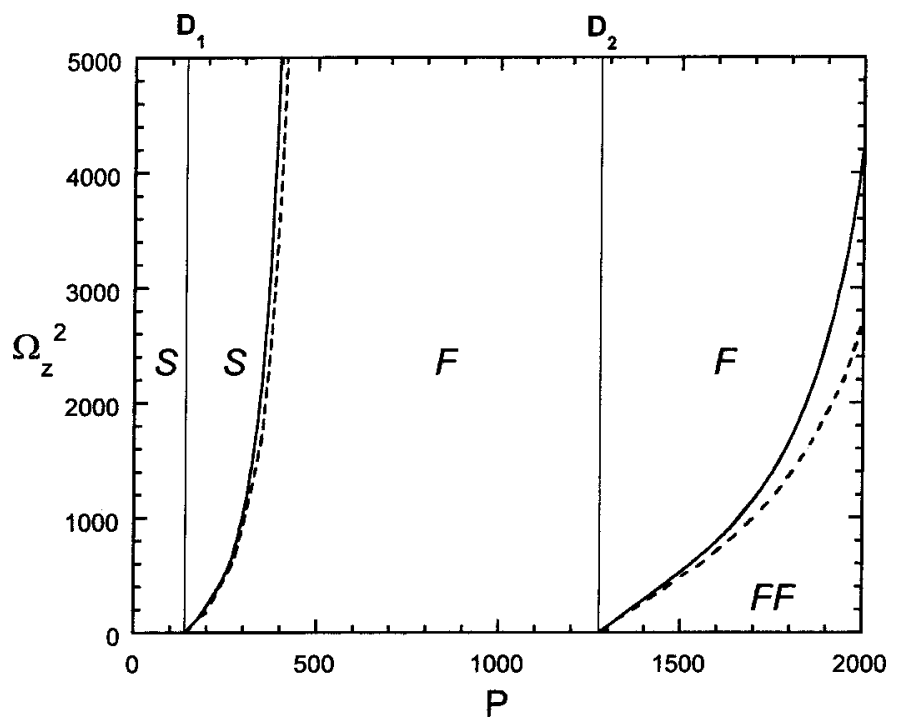

FIG. 12. Validation of the solution methodology based on the Heppler's gyroscopic model (Ref. 5); (- - ) present EGM versus (-----) Heppler's methodology. Stability diagram in the plane $\left(\Omega_{z}^{2}\left[(\mathrm{rad} / \mathrm{s})^{2}\right]\right.$ $-P[N]$.

and quaternary $(D D D D)$ divergence and double flutter $(F F)$, as well as instability regions by mixed, single and double divergence and flutter, i.e., $D F$ and $D D F$, respectively, are featured.

For the case of a solid isotropic beam of cross-sections characterized by $R \neq 1$, the complexity of the stability behavior was illustrated in Ref. 5.

Having in view that, to the best of the authors' knowledge, there are no similar results in the specialized literature based on the thin-walled structural model, the only possible validation would concern the solution methodology used to derive the instability boundaries. In this context, it is possible to apply the present methodology based on the extended Galerkin method (see Ref. 9) to the very simple structural model developed in Ref. 5, where the Ritz method used in conjunction with the eigenfunctions proper to a cantilevered beam have been used. For the data characterizing the gyroscopic system considered in Ref. 5, Fig. 12 displays the instability boundaries obtained via the use of both methodologies, and a good agreement of predictions of the boundaries of stability/instability is revealed.

\section{CONCLUSIONS}

The equations governing the motion of robotic arm systems consisting of a thin-walled elastic beam carrying at its tip a spinning tip rotor loaded by a compressive conservative force have been established.

In this context, the vibrational and stability responses of the considered gyroscopic system have been analyzed, and ways to enhance its behavior, based on the directionality property of the constituent composite material, have been emphasized. The results have also revealed that the system can lose its stability by divergence by the separate actions of $\bar{P}$ and $\bar{\Omega}_{z}$, and by flutter, through the joint action of $\bar{P}$ and $\bar{\Omega}_{z}$. This implies that in the absence of the compressive load, the system cannot lose its stability by flutter.
The results have also revealed that in some cases the gyroscopic effects can stabilize the conservative system.

\section{APPENDIX A: EXPRESSIONS OF THE INERTIA TERMS}

When restricted to the CUS beam configuration the expressions of the inertia terms are

$$
\begin{aligned}
& K_{1}=b_{1} \ddot{u}_{0} ; \quad K_{2}=b_{1} \ddot{v}_{0} ; \quad K_{4}=\left(b_{4}+\delta_{n} b_{14}\right) \ddot{\theta}_{X} ; \\
& K_{5}=\left(b_{5}+\delta_{n} b_{15}\right) \ddot{\theta}_{Y} .
\end{aligned}
$$

In these expressions $b_{i}$ are reduced mass terms defined as

$$
\begin{aligned}
& \left(b_{1}, b_{4}, b_{5}\right)=\oint m_{0}\left(1, Y^{2}, X^{2}\right) d s, \\
& \left(b_{14}, b_{15}\right)=\oint m_{2}\left(\left(\frac{d X}{d s}\right)^{2},\left(\frac{d Y}{d s}\right)^{2}\right) d s,
\end{aligned}
$$

where

$$
\left(m_{0}, m_{2}\right)=\sum_{k=1}^{N} \int_{h_{k-1}}^{h_{(k)}} \rho_{(k)}\left(1, n^{2}\right) d n,
$$

$\rho_{(k)}$ being the mass density of the kth constituent material layer, while $N$ is the total number of layers.

\section{APPENDIX B: EXPRESSIONS OF THE MASS MATRIX $M$, STIFFNESS MATRIX K AND SKEW-SYMMETRIC MATRIX G}

The entries of the mass matrix $\mathbf{M}\left(\equiv \mathbf{M}_{4 \times 4}\right)$ are the following.

$$
\mathbf{M} \equiv\left(m_{i j}\right) \quad \text { Their expression }
$$

$\begin{array}{ll}m_{11} & -\int_{0}^{L}\left(b_{1} \mathbf{U} \mathbf{U}^{T}\right) d Z-\left[m_{R} \mathbf{U} \mathbf{U}^{T}\right]_{Z=L} \\ m_{12}=m_{21} & 0 \\ m_{13}=m_{31} & 0 \\ m_{14} & {\left[m_{R} r_{m} \mathbf{U} \mathbf{Y}^{T}\right]_{Z=L}} \\ m_{22} & -\int_{0}^{L}\left(b_{1} \mathbf{V} \mathbf{V}^{T}\right) d Z-\left[m_{R} \mathbf{V} \mathbf{V}^{T}\right]_{Z=L} \\ m_{23} & {\left[m_{R} r_{m} \mathbf{V} \mathbf{X}^{T}\right]_{Z=L}} \\ m_{24}=m_{42} & 0 \\ m_{32} & {\left[m_{R} r_{m} \mathbf{X} \mathbf{V}^{T}\right]_{Z=L}} \\ m_{33} & -\int_{0}^{L}\left(b_{4}+\delta_{n} b_{14}\right) \mathbf{X} \mathbf{X}^{T} d Z-\left[J_{x x y y}^{R} X X^{T}\right]_{Z=L} \\ m_{34}=m_{43} & 0 \\ m_{41} & {\left[m_{R} r_{m} \mathbf{Y} \mathbf{U}^{T}\right]_{Z=L}} \\ m_{44} & -\int_{0}^{L}\left(b_{5}+\delta_{n} b_{15}\right) \mathbf{Y} \mathbf{Y}^{T} d Z-\left[J_{x x y y}^{R} \mathbf{Y} \mathbf{Y}^{T}\right]_{Z=L}\end{array}$

The entries of the gyroscopic matrix $\mathbf{G}\left(\equiv \mathbf{G}_{4 \times 4}\right)$ different of zero are the following.

\begin{tabular}{cc}
$\mathbf{G} \equiv\left(g_{i j}\right)$ & Their expressions \\
\hline$g_{34}$ & {$\left[3 J_{z z}^{R} \Omega_{z} \mathbf{X Y} \mathbf{Y}^{T}\right]_{Z=L}$} \\
$g_{43}$ & $-\left[3 J_{z z}^{R} \Omega_{z} \mathbf{Y} \mathbf{X}^{T}\right]_{Z=L}$
\end{tabular}


The entries of the stiffness matrix $\mathbf{K}\left(\equiv K_{4 \times 4}\right)$ are

\begin{tabular}{|c|c|}
\hline $\mathbf{K} \equiv\left(K_{i j}\right)$ & Their expression \\
\hline$k_{11}$ & $\begin{array}{l}\int_{0}^{L}\left(a_{44} \mathbf{U} \mathbf{U}^{\prime \prime} T-P \mathbf{U} \mathbf{U}^{\prime \prime} T\right) d Z \\
-\left[a_{44} \mathbf{U} \mathbf{U}^{\prime} T-P \mathbf{U} \mathbf{U}^{\prime}\right]_{Z=L}\end{array}$ \\
\hline$k_{12}=k_{21}$ & -5 \\
\hline$k_{13}$ & $\int_{0}^{L}\left(a_{43} \mathbf{U} \mathbf{X}^{\prime \prime}\right) d Z-\left[a_{43} \mathbf{U} \mathbf{X}^{\prime}\right]_{Z=L}$ \\
\hline$k_{14}$ & $\int_{0}^{L}\left(a_{44} \mathbf{U} \mathbf{Y}^{\prime}\right) d Z-\left[a_{44} \mathbf{U} \mathbf{Y}^{T}\right]_{Z=L}$ \\
\hline$k_{22}$ & $\begin{array}{l}\int_{0}^{L}\left(a_{55} \mathbf{V} \mathbf{V}^{\prime \prime} T-P \mathbf{V} \mathbf{V}^{\prime \prime} T\right) d Z \\
-\left[a_{55} \mathbf{V} \mathbf{V}^{\prime} T-P \mathbf{V} \mathbf{V}^{\prime} T\right]_{Z=L}\end{array}$ \\
\hline$k_{23}$ & $\int_{0}^{L}\left(a_{55} \mathbf{V} \mathbf{X}^{\prime} T\right) d Z-\left[a_{55} \mathbf{V} \mathbf{X}^{T}\right]_{Z=L}$ \\
\hline$k_{24}$ & $\int_{0}^{L}\left(a_{52} \mathbf{V} \mathbf{Y}^{\prime \prime} T\right) d Z-\left[a_{52} \mathbf{V} \mathbf{Y}^{\prime} T\right]_{Z=L}$ \\
\hline$k_{31}$ & $\int_{0}^{L}\left(a_{34} \mathbf{X} \mathbf{U}^{\prime \prime} T\right) d Z-\left[a_{34} \mathbf{X} \mathbf{U}^{\prime} T\right]_{Z=L}$ \\
\hline$k_{32}$ & $-\int_{0}^{L}\left(a_{55} \mathbf{X} \mathbf{V}^{\prime}\right) d Z$ \\
\hline$k_{33}$ & $\int_{0}^{L}\left(a_{33} \mathbf{X} \mathbf{X}^{\prime \prime} T-a_{55} \mathbf{X} \mathbf{X}^{T}\right) d Z-\left[a_{33} \mathbf{X} \mathbf{X}^{\prime} T\right]_{Z=L}$ \\
\hline$k_{34}$ & $\int_{0}^{L}\left(a_{34} \mathbf{X} \mathbf{Y}^{\prime} T-a_{52} \mathbf{X} \mathbf{Y}^{\prime} T\right) d Z-\left[a_{34} \mathbf{X} \mathbf{Y}^{T}\right]_{Z=L}$ \\
\hline$k_{41}$ & $-\int_{0}^{L}\left(a_{44} \mathbf{Y} \mathbf{U}^{\prime} T\right) d Z$ \\
\hline$k_{42}$ & $\int_{0}^{L}\left(a_{25} \mathbf{Y} \mathbf{V}^{\prime \prime} T\right) d Z-\left[a_{25} \mathbf{Y} \mathbf{V}^{\prime} T\right]_{Z=L}$ \\
\hline$k_{43}$ & $\int_{0}^{L}\left(a_{25} \mathbf{Y} \mathbf{X}^{\prime} T-a_{43} \mathbf{Y} \mathbf{X}^{\prime}\right) d Z-\left[a_{25} \mathbf{Y} \mathbf{X}^{T}\right]_{Z=L}$ \\
\hline$k_{44}$ & $\int_{0}^{L}\left(a_{22} \mathbf{Y} \mathbf{Y}^{\prime \prime} T-a_{44} \mathbf{Y} \mathbf{Y}^{T}\right) d Z-\left[a_{22} \mathbf{Y} \mathbf{Y}^{\prime}\right]_{Z=L}$ \\
\hline
\end{tabular}

We should remark that applying an integration by parts in the expressions of $k_{14}$ and $k_{23}$ one obtains, in conjunction with the boundary conditions that $k_{14}$ and $k_{23}$ reduce to

$$
k_{14}=-\int_{0}^{L}\left(a_{44} \mathbf{U}^{\prime} \mathbf{Y}^{T}\right) d Z
$$

and

$$
k_{23}=-\int_{0}^{L}\left(a_{55} \mathbf{V}^{\prime} \mathbf{X}^{T}\right) d Z,
$$

respectively, wherefrom one arrives at the conclusion that the stiffness matrix $\mathbf{K}$ is symmetric.

${ }^{1}$ O. Song, and L. Librescu, "Anisotropy and Structural Coupling on Vibration and Instability of Spinning Thin-Walled Beams," J. Sound Vib. 204(3), 477-494 (1997).

${ }^{2}$ O. Song and L. Librescu, "Modeling and Vibration of Pretwisted Spinning Composite Thin-Walled Beams," Proceedings of the 38th AIAA/ASME/ ASCE/AHS/ASC, Structures, Structural Dynamics, Materials Conference and Exhibition and AIAA/ASME/AHS/Adaptive Structures Forum, Part I, AIAA 97-1091, pp. 312-322, Kissimmee FL, 7-10 April 1997.

${ }^{3}$ G. M. T. D'Eleuterio and P. C. Hughes, "Dynamics of Gyroelastic Spacecraft," J. Guid. Control Dyn. 10(4), 401-405 (1987).

${ }^{4}$ K. Yamanaka, G. R. Heppler, and K. Huseyin, "Stability of Gyroelastic Beams," AIAA J. 34(6), 1270-1278 (1996).

${ }^{5}$ K. Yamanaka, G. R. Heppler, and K. Huseyin, "The Stability of a Flexible Link with a Tip Rotor and a Compressive Tip Load," IEEE Trans. Rob. Autom. 11(6), 882-886 (1995).

${ }^{6}$ O. Song and L. Librescu, "Free Vibration of Anisotropic Composite ThinWalled Beams of Closed Cross-Section Contour," J. Sound Vib. 167(1), 129-147 (1993).

${ }^{7}$ L. Librescu, L. Meirovitch, and O. Song, "Refined Structural Modeling for Enhancing Vibrational and Aeroelastic Characteristics of Composite Aircraft Wings," Rech. Aerosp. 1, 23-35 (1996).

${ }^{8}$ L. Librescu, O. Song, and H. D. Kwon, "Vibration and Stability of Gyroelastic Beams Via Smart Materials Technology," Smart Structures, NATO Science Series, 3. High Technology-Vol. 65, edited by J. Holnicki-Szulc and J. Rodellar (Kluwer Academic, Dordrecht, 1999), pp. $163-172$.

${ }^{9}$ L. Librescu, L. Meirovitch, and S. S. Na, "Control of Cantilever Vibration via Structural Tailoring and Adaptive Materials," AIAA J. 35(8), 13091315 (1997).

${ }^{10}$ P. C. Hughes, Spacecraft Attitude Dynamics (Wiley, New York, 1986).

${ }^{11} \mathrm{~K}$. Huseyin, Vibrations and Stability of Multiple Parameters Systems (Noordhoff, Alphen Aan Den Rijn, The Netherlands, 1978). 